Aims. Assess how current practice reflects recommendations from the National Confidential Enquiry into Patient Outcome and Death (NCEPOD) Treat as One: bridging the gap between mental and physical healthcare report (January 2017).

Develop template for electronic documentation of liaison psychiatry reviews and implement for trial period.

Re-audit after trial period to assess for change in quality of documentation.

Background. The John Radcliffe Hospital (JR) is a tertiary centre and has a large liaison psychiatry department with 14 consultants. Patient reviews by the liaison team are documented using a blank note type, on an electronic system used by all specialties within the hospital trust. The NCEPOD Treat as One report makes recommendations for the content of documentation of liaison psychiatry reviews which aim to improve communication between specialties.

Method. 86 patients referred to liaison psychiatry at the JR in September 2018 were randomly selected. Four liaison psychiatry consultants appraised the quality of documentation of anonymized reviews by consultant colleagues. The audit tool was a questionnaire containing 12 questions developed by the four consultants based on the NCEPOD Treat as One report. Data were collated from these questionnaires. The template for electronic documentation was developed to reflect the report recommendations and after discussion with the liaison psychiatry team. The template has been implemented and is used for all initial patient reviews.

Result. The 12 questions of the audit tool can be divided into two groups: assessment and management. As part of the assessment, the majority of reviews included a primary diagnosis (77.9\%) and reason for referral (66.3\%). Other aspects of the assessment were documented in the minority of reviews: mental capacity (19.8\%), need for DOLS (2.3\%), risks (27.9\%) and risk management $(7 \%)$. Regarding the management, the majority of reviews included: clear plan with numbered/bullet points (61.6\%), medication changes (51.4\%), useful plan (73\%) and answered the reason for referral $(69.8 \%)$. Other aspects of the management were documented in the minority of reviews: each action point assigned (47.7\%) and non-medical MDT advice $(18.6 \%)$.

Conclusion. The main area for improvement in documentation of assessment agreed by the liaison team is risk. The main areas agreed for improvement in documentation of management are medication changes, assigning action points to individuals, and including advice for non-medical MDT members. The next step is re-audit, planned for March 2020.

\section{Improving access to the physical health clinic in a community first-episode psychosis service}

\author{
Zena Tansley-Ahmed ${ }^{1 \star}$ and Wei Han Lim $^{2}$ \\ ${ }^{1}$ Imperial College Healthcare NHS Trust and ${ }^{2}$ Central and North \\ West London NHS Foundation Trust \\ ${ }^{*}$ Corresponding author.
}

doi: 10.1192/bjo.2021.598

Aims. Physical health outcomes are poor for patients with severe mental illness as demonstrated by the significant mortality gap present globally.[1] Access to and engagement with care is a key factor underpinning this disparity.[2] The Early Intervention in Psychosis service works with young people from 14-35 experiencing a first episode of psychosis in the community. Within the service, difficulties in engagement have been reflected in the high 'no-show' rates observed in the Foundation Year 2 trainee doctor-led physical health clinic. This quality improvement project aimed to reduce the 'did not attend' (DNA) rate in the physical health clinic by $20 \%$ in order to improve patient outcomes, particularly in the context of their physical health.

Method. The project took place between September and November 2020, over the course of 10 weeks. A driver diagram was constructed to identify key influencing factors and subsequent change ideas. In order to implement each of these changes, three cycles within the Plan, Do, Study, Act (PDSA) ramp framework were completed. These consisted of phone reminders within 48 hours of appointments, a teaching session for staff alongside the distribution of an accompanying information leaflet and increased flexibility in clinic times with opportunistic appointments. The change ideas were cumulative with each cycle lasting a duration of seventeen days.

Result. The baseline DNA rate was calculated based on the preceding month and found to be $55 \%$. Following cycle one of the project, there was a significant reduction in DNA rates to $30 \%$ although this remained relatively stable at $33 \%$ after cycle two. By the end of cycle three when all interventions had been introduced, the DNA rate had dropped to $22 \%$. As such, a total drop in DNA rate of over 30\% was achieved which surpassed the initial aim of the project.

Conclusion. The outcomes of this project demonstrate that the introduction of even simple measures can lead to positive change. Successful implementation of these changes requires teamwork and a culture of openness and flexibility. Feedback from team members, particularly care coordinators, also indicated better resulting engagement of clients with the service overall, suggesting potential for both improved mental and physical health outcomes. Next steps for this project may involve not only continued implementation of established changes but also service user input and scope for virtual consultations particularly in light of current COVID-19 restrictions.

\section{Confidentiality at the interface of an adolescent psychotherapy service}

Rhianne Thomas*, Lawrence Congdon and Sheva Habel

Tavistock and Portman NHS Foundation Trust

${ }^{\star}$ Corresponding author.

doi: 10.1192/bjo.2021.599

Aims. Aims included to explore how, within a London trust, staff at the interface between patients, relatives and access to services view their understanding of confidentiality, and to determine ways to improve knowledge if needed.

Background. Confidentiality is essential to the trust and development of clinician-patient relationships. National policies set guidance on how confidential information should be recorded, secured and shared. However, confidentiality breaches are reportedly common within health professions. Working with adolescent patient groups brings additional issues regarding confidentiality. Care-givers who contact services, often desiring containment, may experience a sense of uncertainty when confidentiality policy prevents details being shared about a young person's clinical experience.

Method. Stakeholders were identified from the multidisciplinary team, with a collaborative rather than 'top-down' approach. Administrators in patient-facing roles were surveyed to ascertain current understanding and frequency of involvement in confidentiality issues. Based on feedback, a flowchart prompt was designed, ensuring it reflected best practice. Qualitative and quantitive data were collected before and after a two month implementation period. 
Result. All respondents $(\mathrm{n}=10)$ dealt with confidentiality issues at work, with $50 \%$ experiencing issues daily. $33 \%$ respondents did not feel confident dealing with confidentiality queries at work. The majority (60\%) had received confidentiality training, but all respondents thought extra information would be useful. Of possible interventions, 70\% supported a flowchart. Following an implementation period, 100\% respondents re-surveyed agreed they felt confident dealing with issues related to confidentiality at work. The majority of respondents had used the flowchart and found it useful (83\%). Qualitative data gathered suggested rollingout the project elsewhere.

Conclusion. A lack of confidence surrounding issues with confidentiality, including information sharing, was identified. This can negatively impact patient engagement and delivery of care. The introduction of the confidentiality flowchart demonstrated improved understanding of, and confidence in, patient confidentiality issues. The small sample size means there are limitations in extrapolating findings to wider contexts. However, it is likely that more confidentiality training and practical information for NHS staff at the interface between patients, clinicians and services would reduce the risk of confidentiality breaches and reinforce positive relationships with services.

Improving patient access to medication information: a quality improvement project on patient-centred prescribing

Anna Todd* and Rosy Blunstone

Bethlem Royal Hospital, South London and Maudsley NHS

Foundation Trust

${ }^{*}$ Corresponding author.

doi: 10.1192/bjo.2021.600

Aims. Ward rounds are sometimes the only opportunity for patients to discuss medication. Patient and professional feedback on an acute male inpatient ward in South London highlighted a demand for more medication information outside the formal ward round setting. We aimed to have $100 \%$ of patients meet our criteria for "Patient-Centred Prescribing" on the ward by March 2021. To fulfil criteria, all patients are offered: (1) ward round discussion, (2) written patient information leaflets (PILs), (3) informal discussion groups, all regarding medication.

The principles of this quality improvement project (QIP) were drawn from definitions of patient-centred care and standards of good practice; patients should have access to a variety of information formats, relevant to the individual, and the knowledge gained empowers patients.

Patient experience data revealed that $30 \%$ of clients answered passively to the question, "Do you feel involved in your care?" We hypothesized that medication discussion groups positively impact patients' wellbeing, by providing a safe space that facilitates conversation surrounding medication issues.

Method. We conducted weekly audits on patients whose admission duration was $>7$ days, and recorded fulfilment of the above criteria. At week 1, we introduced a program of weekly medication discussion groups led by members of the wider multidisciplinary team covering a broad topic range. At week 6 , we developed a rolling rota of the discussion groups and posters were displayed in advance. At week 14, all patients were offered PILs through a 1:1 interaction and this continued as routine practice. Medication discussion group feedback was obtained via questionnaires and "The Blob Tree", a psycho-emotional assessment tool commonly used in healthcare settings.
Result. In 19 weeks, the median percentage of patients who fulfilled our criteria for Patient-Centred Prescribing was 92.86\%. After 11 medication discussion groups, $79.3 \%$ of questionnaire responders wanted further sessions. $88 \%$ of "The Blob Tree" responses collected inferred a positive emotional response after the group discussions and half of those noticed an improvement in their emotional state. Conclusion. This QIP was overall a success; it fulfilled a requirement to meet good standards in information sharing and became embedded in the fabric of the ward, continuing to run as part of the activities program. It demonstrated the impact of education on patients' mental wellbeing through empowerment and peer support. As a by-product it established multidisciplinary connections and improved therapeutic relationships. Challenges included patient engagement secondary to acute mental illness or negative symptoms and maintaining project momentum following a COVID-19 outbreak.

\section{On-line memory clinic - piloting a hybrid model}

\section{Rahul Tomar}

Logandene, Hertfordshire Partnership University NHS Foundation NHS Trust

doi: 10.1192/bjo.2021.601

Aims. Quality improvement project was undertaken to reorganise memory clinic to incorporate both virtual and in-person consultation (Hybrid Virtual model), as depicted in the following model: Method. Tele triage conducted to ascertain information from patient and carer. This reduced time for face to face assessment.

Nurse did face to face assessment to complete cognitive test (Addenbrooke's Cognitive Examination III) \& carer completed Bristol Activities of Daily Living scale. Nurse would also do BP, $\mathrm{PR}$, oxygen sats \& temp.

Nurse discussed the assessment with the consultant (who is in the inpatient unit) on line using MS TEAMS

Consultant would then see patient on line, confirm diagnosis, answer questions, give information on medication and post prescription (if required)

Feedback was collected using Telehealth Satisfaction questionnaire Result. Hybrid remote memory clinic was started on 29/09/20. A total of 37 patients were seen in this clinic by 31/01/21.

Collected feedback from 21 patients was generally positive Information provided on video consultation prior to assessment -18 reported it as excellent

How well you privacy was respected - 21 reported it as excellent. Information you received on the treatment -18 reported it as excellent

Conclusion. The hybrid remote memory clinic was more effective than telephone consultation or on line only consultation as it was -

Easy to establishing rapport

Physical examination could be performed

Digital literacy was no longer a limiting factor

Prescribing medication was slightly more difficult but possible

Audit of follow-up within 7 days on discharge from the mental health unit, Forth Valley Royal Hospital

Matthew Turner ${ }^{1 *}$, Shaun Love ${ }^{2}$, Fergus Douds ${ }^{1}$

and Anyssa Zebda ${ }^{1}$

${ }^{1}$ Forth Valley Royal Hospital and ${ }^{2}$ NHS Greater Glasgow and Clyde ${ }^{\star}$ Corresponding author.

doi: 10.1192/bjo.2021.602 